\title{
Inserting Points Uniformly at Every Instance
}

\author{
Sachio Teramoto ${ }^{1}$, Tetsuo Asano ${ }^{1}$, Benjamin Doerr ${ }^{2}$, and Naoki Katoh ${ }^{3}$ \\ 1 School of Information Science, Japan Advanced Institute of Science and Technology (JAIST), 1-1 Asahidai, Nomi, \\ Ishikawa, 923-1292 Japan, \{s-teramo, t-asano\}@jaist.ac.jp \\ 2 Max Planck Institute für Informatik, Saarbrücken, Germany, doerr@mpi-sb.mpg.de \\ 3 Graduate School of Engineering, Kyoto University, Kyoto-Daigaku-Katsura, Nishigyoku, Kyoto, 615-8540, Japan, \\ naoki@archi.kyoto-u.ac.jp
}

\begin{abstract}
A problem of arranging $n$ points as uniformly as possible, which is equivalent to that of packing $n$ equal and non-overlapping circles in a unit square, is frequently asked. In this paper we generalize this problem in such a way that points be inserted one by one with uniformity preserved at every instance. Our criteria on uniformity is to minimize the gap ratio (which is the maximum gap over the minimum gap) at every point insertion. We present a linear time algorithm for finding an optimal $n$-point sequence with the maximum gap ratio bounded by $2^{\lfloor n / 2\rfloor /(\lfloor n / 2\rfloor+1)}$ in the 1-dimensional case. We describe how hard the same problem is for a point set in the plane and propose a local search heuristics for finding a good solution.
\end{abstract}

\section{Introduction}

Circle packing problem to place $n$ equal and non-overlapping circles in a unit square is one of important geometric optimization problems with a number of applications and has been intensively investigated [6,12-14]. It is well known that the circle packing problem is equivalent to that of placing $n$ points in a unit square in such a way that the minimum pairwise distance is maximized. This problem seems to be computationally hard. In fact, no optimal solution is known for relatively large value of $n$, say $n>100$.

A problem to be considered in this paper is a generalization of the point arranging problem. That is, the point arranging problem requires arrangement of $n$ points in a unit square such that those points are distributed as uniformly as possible. In our problem we want to insert $n$ points one by one in such a way that uniformity is achieved at every insertion of a point. Since solutions to the point arranging problems are different for different values of $n$, it is impossible to derive a good point sequence from a set of those optimal solutions even if optimal solutions are available. It should be noted that a subsequence of an optimal point sequence is not optimal. In other words, there is no incremental algorithm for constructing an optimal point sequence.

Another difficulty is how to define uniformity. We could measure uniformity by the minimum pairwise distance as before, but it is not appropriate for our problem because once we have a close point pair it determines the quality of point distribution (uniformity) until a closer point pair is generated. Another possible measure is one based on discrepancy theory. In the measure we take a simple geometric shape $R$ and count how many points are contained in $R$ while moving $R$ all over the unit square. The uniformity is measured by the difference between the largest and smallest counts for all possible sizes of the shape. A serious disadvantage of the measure is computational hardness.

We define uniformity of point distribution using not only closest point pairs but also largest empty circles. Our criterion is to minimize the gap ratio, which is the maximum gap (diameter of a largest empty circle) over the minimum gap (the minimum pairwise distance). One advantage of this definition is extendibility to higher dimensions since those gaps can be defined in any dimension.

A formal definition of our problem is as follows:

This problem is closely related to an industrial application on digital halftoning, which is a technique to convert continuous-tone images into binary images for printing. One of most popular methods for halftoning is Dithering which binarizes an images using a threshold matrix called dither matrix. The quality of output images heavily depends on the matrix. A target there is an $n \times n$ matrix containing 
integers from 0 through $n^{2}-1$ in such a way that elements up to $i$ are uniformly distributed for each $i=$ $1,2, \ldots, n^{2} / 2$. Such a matrix is similar to a dither matrix called blue-noise mask $[11,18]$. Combinatorial approaches are also found for the problem, see e.g., [1,2,4,7,17].

Problem Statement Let $\mathbb{S}^{d}$ be the unit square in the $d$-dimensional space $\mathbb{R}^{d}$ and $P=\left(p_{1}, \ldots, p_{n}\right)$ be a $n$-point sequence contained in $\mathbb{S}^{d}$. We insert $p_{1}, p_{2}, \ldots, p_{n}$ in order. For each subsequence $P_{i}=$ $\left(p_{1}, \ldots, p_{i}\right), i=1,2, \ldots, n$, we define the current point set as $S_{i}:=\left\{p_{1}, \ldots, p_{i}\right\} \cup S_{0}$, where $S_{0}$ is the set of the $2^{d}$ corner points of $\mathbb{S}^{d}$, the maximum gap as $G_{i}:=\max _{p \in \mathbb{S}^{d}} \min _{q \in S_{i}} 2 d(p, q)$, the minimum gap as $g_{i}:=\min _{p, q \in S_{i}, p \neq q} d(p, q)$, where $d(\cdot, \cdot)$ is the Euclidean distance, and the $i$-th gap ratio as $r_{i}:=G_{i} / g_{i}$. The maximum gap and the minimum gap imply the diameter of the largest empty ball and the minimum pairwise distance, respectively.

For a point sequence $P$, we define the maximum gap ratio as $R_{P}:=\max _{i} r_{i}$. For a fixed integer $n$, we denote $R_{n}$ the optimal gap ratio for any $n$-point sequence,

$$
R_{n}:=\min \left\{R_{P} \mid P \text { is any } n \text {-point sequence contained in } \mathbb{S}^{d}\right\} \text {. }
$$

Then, given $d$ and $n$, we want to find an $n$-point sequence $P$ contained in $\mathbb{S}^{d}$ such that $P$ minimizes the maximum gap ratio of itself. More formally, we formulate our problem as follows.

\section{Problem 1:}

Input: Integers $d$ and $n$.

Ensured: Compute an optimal $n$-point sequence $P$ in $\mathbb{S}^{d}$ that achieves $R_{P}=R_{n}$.

Our contribution We start with a simple greedy algorithm called incremental Voronoi insertion in Section 2. The Voronoi insertion generates a point sequence $P$ with $R_{P} \leq 2$. According to this algorithm, one can extend this result into any higher-dimension and increase the size of point sequence without limit.

In Section 3, we present a preliminary result for Problem 1, that is, we give a linear time algorithm for finding a $n$-point sequence with the maximum gap ratio bounded by $2^{\lfloor n / 2\rfloor /(\lfloor n / 2\rfloor+1)}$ in the 1-dimensional unit square $\mathbb{S}^{1}$. We also show that the bound is optimal, hence, our algorithm concludes in the 1-dimensional case.

In Section 4, we consider the planar case, and show a few properties for the optimal point sequence. It may be difficult to solve analytically, hence, we design simple two heuristic algorithms to find a point sequence whose the maximum gap ratio is smaller than that of the point sequence generated by incremental Voronoi insertion. Since Voronoi insertion gives an upper bound, our next goal is the following:

\section{Problem 2:}

Input: Integers $d$ and $n$.

Output: Whether there exists an $n$-point sequence $P$ contained in $\mathbb{S}^{d}$ such that $R_{P}<2$.

We describe implementation of our heuristic algorithm and show our experimental results on a point sequence with maximum gap ratio strictly less than that of Voronoi insertion.

\section{Simple Greedy Algorithm}

We present a simple greedy algorithm for inserting points uniformly called incremental Voronoi insertion. In this algorithm, we maintain a set of vertices which are either Voronoi vertices of a Voronoi diagram for a set of points already inserted or intersections between Voronoi edges and cube surfaces. We evaluate each such vertex by the distance to the nearest point (site) and choose the one of the largest such distance as the next point to be inserted. 
Define a point set $S_{i}^{d}=\left\{\left(x_{1}, x_{2}, \ldots, x_{d}\right) \mid\right.$ exactly $i$ coordinates are either 0 or 1 and the remaining coordinates are $1 / 2\}$ for $i<d$. For example, we have

$$
\begin{aligned}
& S_{0}^{3}=\{(1 / 2,1 / 2,1 / 2)\}, \\
& S_{1}^{3}=\{(*, 1 / 2,1 / 2),(1 / 2, *, 1 / 2),(1 / 2,1 / 2, *)\}, \\
& S_{2}^{3}=\{(*, *, 1 / 2),(*, 1 / 2, *),(1 / 2, *, *)\},
\end{aligned}
$$

where $*$ indicates 0 or 1 , that is, $(*, 1 / 2,1 / 2)$ represents $(0,1 / 2,1 / 2),(1,1 / 2,1 / 2)$.

The first point to be inserted must be the unique element of $S_{0}^{d}$, i.e., $(1 / 2,1 / 2, \ldots, 1 / 2)$. Then, we insert points in the set $S_{1}^{d}$ one by one, and continue to points in $S_{2}^{d}, S_{3}^{d}, \ldots, S_{d-1}^{d}$. Suppose we have inserted all the points in $S_{0}^{d}, S_{1}^{d}, \ldots, S_{d-2}^{d}$ and we are now inserting the first point $p_{j}=(0,0, \ldots, 0,1 / 2)$ in the set $S_{d-1}^{d}$. The point $p_{j}$ is the mid-point of a cube edge by the definition. Thus, the minimum pairwise distance is $1 / 2$, that is, the minimum gap is $1 / 2$. Since this is the first point located on a cube edge, the empty ball centered at the next point $(0,0, \ldots, 0,1,1 / 2)$ that passes through the two points $(0,0, \ldots, 0,0)$ and $(0,0, \ldots, 0,1)$ remains empty. In fact, this ball is the largest empty ball. Its diameter is obviously 1 . Therefore, the ratio is exactly 2 after the point.

We can also show that the maximum ratio before inserting this point is less than 2 and the maximum ratio after inserting the point until the very last point of $S_{d-1}^{d}$ is at most 2 . When we have inserted all the points in $S_{0}^{d}, S_{1}^{d}, \ldots, S_{d-1}^{d}$, we can continue the same process again for $2^{d}$ sub-cubes in a recursive fashion. Thus, we can conclude that the above-mentioned approximation algorithm achieves the maximum ratio 2 .

\section{1-dimensional problem}

Our domain here is a unit interval $[0,1]$. The two extremal points 0 and 1 are assumed to be inserted in advance. We present a simple linear time strategy better than the incremental Voronoi insertion. Moreover, we show that the strategy is in fact optimal.

\subsection{Lower bound on $R_{n}$}

We first estimate the lower bound of $R_{n}$ for an $n$-point sequence. Let $P=\left(p_{1}, p_{2}, \ldots, p_{n}\right)$ be a finite sequence of $n$ points in the unit interval $[0,1]$ such that $p_{i} \neq p_{j}$ whenever $i \neq j$. For $i=0, \ldots, n$, the points $p_{1}, \ldots, p_{i}$ partition the unit interval into $i+1$ intervals of lengths $m_{1}^{i}, m_{2}^{i}, \ldots, m_{i+1}^{i}$. Without loss of generality we may assume that $m_{j}^{i} \geq m_{j+1}^{i}$ for all $i, 0 \leq i \leq n$ and $j, 1 \leq j \leq i$. Then, the maximum and minimum gaps are given by $m_{1}^{i}$ and $m_{i+1}^{i}$, respectively. Hence, the ratio $R_{P}$ for the sequence $P$ is

$$
R_{P}:=\max _{1 \leq i \leq n} \frac{m_{1}^{i}}{m_{i+1}^{i}}
$$

Put $M^{i}=\left\{m_{1}^{i}, \ldots, m_{i+1}^{i}\right\}$ and regard it as a multi-set (i.e., it may contain elements more than once). Clearly, $M^{i+1}$ is obtained from $M^{i}$ by replacing one element from $M^{i}$ by two which add up to the first one. The following lemma states that if $R_{P} \leq 2$, then this replaced element is always the largest.

Lemma 1. If $R_{P} \leq 2$, then for each $i=0, \ldots, n-1$ there are $a, b \in[0,1]$ such that $m_{1}^{i}=a+b$ and $M^{i+1}=\left\{m_{2}^{i}, \ldots, m_{i+1}^{i}, a, b\right\}$ (as multi-set) and one of $a$ and $b$ is a smallest element of $M^{i+1}$.

Proof. Assume that $M^{i+1}=M^{i} \backslash\left\{m_{j}^{i}\right\} \cup\{a, b\}$ for some $j, 1 \leq j \leq i+1$ such that $m_{j}^{i}<m_{1}^{i}$ and $a+b=m_{j}^{i}$. W.l.o.g., let $b \leq a$. Then, $b \leq \frac{1}{2} m_{j}^{i}<\frac{1}{2} m_{1}^{i}$ and hence $R_{P} \geq m_{1}^{i+1} / b=m_{1}^{i} / b>2$. If both $a$ and $b$ are greater than $m_{i+1}^{i}$, then again $R_{P} \geq m_{1}^{i} / m_{i+1}^{i}=(a+b) / m_{i+1}^{i}>2 m_{i+1}^{i} / m_{i+1}^{i}=2$.

Note, however, that a priori we do not know that both $a$ and $b$ are not larger than $m_{i+1}^{i}$. 
Lemma 2. Given an integer $n \geq 1$, the lower bound of $R_{n}$ is $2^{\lfloor n / 2\rfloor /(\lfloor n / 2\rfloor+1)}$.

Proof. Assume that $R_{P} \leq 2$ for an $n$-point sequence. Let first $n$ be even. Let $j, 1 \leq j \leq \frac{n}{2}+1$ be such that $m_{j}^{n / 2} \in M^{n}$. Such a $j$ exists, since at most $n / 2$ of the elements in $M^{n / 2}$ are replaced in the sequel from $M^{n / 2}$ to $M^{n}$. We have

$$
\frac{m_{1}^{n / 2}}{m_{j}^{n / 2}} \leq \frac{m_{1}^{n / 2}}{m_{n / 2+1}^{n / 2}} \leq R_{P}
$$

Also, for each $n / 2 \leq i \leq n-1$, we have $R_{P} \geq m_{1}^{i+1} / m_{i+2}^{i+1} \geq m_{1}^{i+1} / m_{j / 2}^{i}$ by Lemma 1 . Since $m_{j}^{n / 2} \in M^{n}$,

$$
R_{P} \geq \frac{m_{1}^{n / 2}}{m_{j}^{n / 2}} \geq \frac{m_{1}^{n / 2}}{m_{1}^{n}}=\prod_{i=n / 2}^{n-1} \frac{m_{1}^{i}}{m_{1}^{i+1}}=\prod_{i=n / 2}^{n-1} \frac{m_{1}^{i}}{m_{i+2}^{i+1}}\left(\frac{m_{1}^{i+1}}{m_{i+2}^{i+1}} \geq\left(\frac{2}{R_{P}}\right)^{n / 2}\right.
$$

We conclude $R_{P} \geq 2^{(n / 2) /(n / 2+1)}$. For $n$ odd, let $P^{\prime}=\left(p_{1}, \ldots, p_{n-1}\right)$. Then, $R_{P} \geq R_{P^{\prime}}$ by definition and $R_{P^{\prime}} \geq 2^{\lfloor n / 2\rfloor /(\lfloor n / 2\rfloor+1)}$ by the above. So, $R_{n} \leq 2^{\lfloor n / 2\rfloor /(\lfloor n / 2\rfloor+1)}$. This completes the proof of Lemma 2 .

So, we have obtained the lower bound of $R_{n}$ for $n$-point sequences. Now, what remains is for the 1-dimensional case is to give an algorithm for computing an optimal point sequence $P^{*}$.

First consider the following algorithm suggested in the lower bound proof.

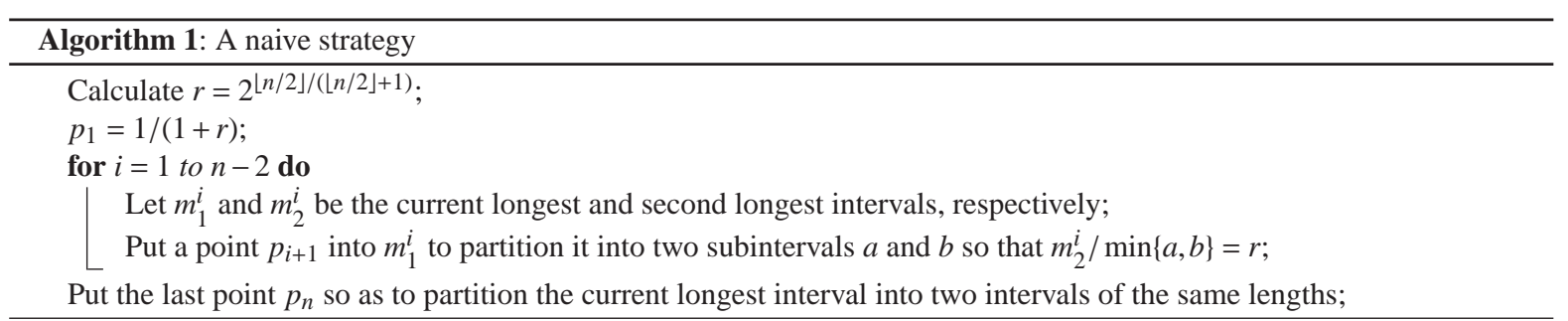

This strategy always puts a point $p_{i}$ so that the gap ratio is equal to $2^{\lfloor n / 2\rfloor /(\lfloor n / 2\rfloor+1)}$ for each $i$. If it is possible then the sequence obtained is optimal since its bound coincides with the lower bound. The strategy implicitly assumes that the smaller one of the new subintervals has the minimum length among current intervals. Unfortunately, it is impossible to keep the ratio. The reason is as follows. Let $a_{i}$ and $b_{i}\left(a_{i}>b_{i}\right)$ be new subintervals generated after the $i$-th insertion. Then, $M^{1}=\left\{a_{1}, b_{1}\right\}=\left\{\frac{r}{r+1}, \frac{1}{r+1}\right\}$ and $M^{2}=\left\{b_{1}, a_{2}, b_{2}\right\}=\left\{\frac{1}{r+1}, \frac{r-1}{r}, \frac{1}{r(r+1)}\right\}$. We insert $p_{3}$ into $M^{2}$. Note that the maximum interval length in $M^{2}$ depends on the number of points to be inserted. If $b_{1} \geq a_{2}$, (the case of $r \leq \frac{1+\sqrt{5}}{2}$ ), then $b_{3}=\frac{a_{2}}{r}=\frac{r-1}{r^{2}}$ and $a_{3}=\frac{1}{r+1}-b_{3}=\frac{1}{r^{2}(r+1)}$. Since $a_{3}<b_{3}$ for $r>\sqrt{2}, r_{3}=a_{2} / a_{3}=r\left(r^{2}-1\right)>R_{n}$. This suggests that if $n$ is large enough, say $n>3$, the assumption of above strategy does not hold. On the other hand, if $b_{1}<a_{2}$ (the case of $r>\frac{1+\sqrt{5}}{2}$ ), then $r_{2}=\frac{a_{2}}{b_{2}}=r^{2}-1$, and $2<R_{n}^{2}-1$ for $n \geq 8$. Therefore, we cannot obtain an optimal point sequence $P^{*}$ by the above strategy.

Observation 1. Gap ratios for the first $n-1$ points should be strictly less than $2^{\lfloor n / 2\rfloor /(\lfloor n / 2\rfloor+1)}$, and moreover, these ratios are never determined until the last interval is fixed.

This Observation 1 suggests that an optimal point sequence of length $n$ should be determined in a bottom-up fashion, that is, from the last interval to the first one.

\subsection{An optimal point insertion strategy}

A rough sketch of our strategy is as follows. Let $\left(p_{1}, p_{2}, \ldots, p_{n}\right)$ be a point sequence to be inserted in the unit interval $x_{1}=[0,1]$. We maintain all intervals generated during $n$ insertions, and we denote by 
$x_{j}$ the interval induced by $p_{\lfloor n / 2\rfloor}$. Hereafter, we denote the $j$-th interval by $x_{j}$, and unify $x_{j}$ and its length $\left|x_{j}\right|$. Each point $p_{i}, i=1, \ldots, n$, is inserted into the current largest interval $x_{i}$ to split it into two new subintervals $x_{2 i}$ and $x_{2 i+1}$ with $x_{2 i}+x_{2 i+1}=x_{i}$. An important observation here is that we can determine the point $p_{i}$ so that it results in a sorted sequence $\left(x_{i+1}, x_{i+2}, \ldots, x_{2 i}, x_{2 i+1}\right)$ of intervals in the decreasing order of their lengths. The process is terminated when the last point $p_{n}$ is inserted to have a sequence $\left(x_{n+1}, x_{n+2}, \ldots, x_{2 n+1}\right)$.

Now, let us describe how to determine the point sequence. It is divided into two subsequences at $k=\lfloor n / 2\rfloor$. For the first half $\left(p_{1}, \ldots, p_{k}\right)$, the current longest interval $x_{i}$ is unevenly partitioned into the new two subintervals $x_{2 i}$ and $x_{2 i+1}$, so that $x_{2 i}>x_{2 i+1}$ and $x_{i}=x_{2 i}+x_{2 i+1}$. Since we are trying to achieve a ratio strictly less than 2 , the ratio $x_{i+1} / x_{2 i+1}$ must be strictly less than 2 . For the remaining points $\left(p_{k+1}, \ldots, p_{n}\right)$, the current longest interval $x_{i}$ is partitioned evenly into two new subintervals $x_{2 j}$ and $x_{2 j+1}$ so that $x_{2 j}=x_{2 j+1}=x_{j} / 2$ and $x_{j+1} / x_{2 j+1}$ is strictly less than 2 , or equal to $R_{n}$. This is because the intervals $x_{2 i}$ and $x_{2 i+1}, i=k+1, \ldots n$, will never be subdivided during the remaining insertion. Since minimum gaps are maximized by evenly partitioning, it minimizes the maximum gap ratios.

More concretely, we first compute the target ratio $R_{n}=2^{k /(k+1)}$ where $k=\lfloor n / 2\rfloor$, and a magic number $y_{1}=\left(2^{l-k}+2 \sum_{i=2}^{k+1} \frac{R_{n}^{i-1}}{2^{i-1}}\right)^{-1}$, where $l=\lceil n / 2\rceil$. Then, we fix the last $2 k+2$ interval;

$$
\begin{gathered}
x_{2 l}=x_{2 l+1}=y_{1} \quad \text { if } n \text { is odd, } \\
x_{2 l+1}=y_{1} \quad \text { if } n \text { is even, } \\
x_{2(l+1)}=x_{2(l+1)+1}=\frac{R_{n}}{2} y_{1}, \\
x_{2(l+2)}=x_{2(l+2)+1}=\left(\frac{R_{n}}{2}\right)^{2} y_{1}, \\
\vdots \\
x_{2(l+k)}=x_{2(l+k)+1}=\left(\frac{R_{n}}{2}\right)^{k} y_{1} .
\end{gathered}
$$

The remaining intervals can be determined so that $x_{i}=x_{2 i}+x_{2 i+1}, i=k, k-1, \ldots, 2,1$. This strategy can be summarized in the following pseudo code.

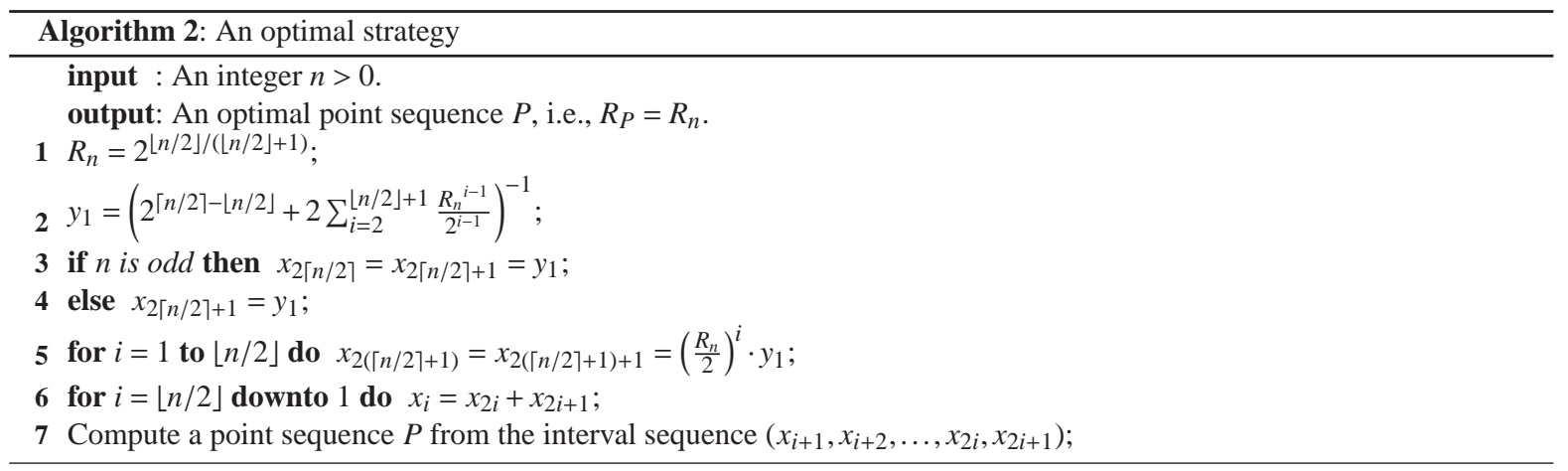

\subsection{Configuration tree}

Before showing the optimality and correctness of our strategy, we introduce a notation, in order to simplify the arguments for proof. For any point sequence $P$, every $i$-th insertion can be represented by a rooted tree structure with size $2 i+1$, where the root is the unit segment $x_{1}$. For $i$-th insertion, each node corresponds to an interval generated by $p_{1}, \ldots, p_{i}$, and an interval sequence $\left(x_{i+1}, \ldots, x_{2 i+1}\right)$ is obtained 
form the leaves in old order. The two nodes are connected by an edge, if one node is induced from the other node by inserting a point. More formally, there are two edges start for the node of $x_{i}, i=1, \ldots,\left\lfloor\frac{n}{2}\right\rfloor$, to the nodes of $x_{2 i}$ and $x_{2 i+1}$, respectively. This implies that every inner node is already subdivided. Figure 1 shows an example of the configuration tree for $n=4$. The grey nodes are leaf nodes. We can see that the intervals corresponding to leaf nodes subdivide the unit segment.

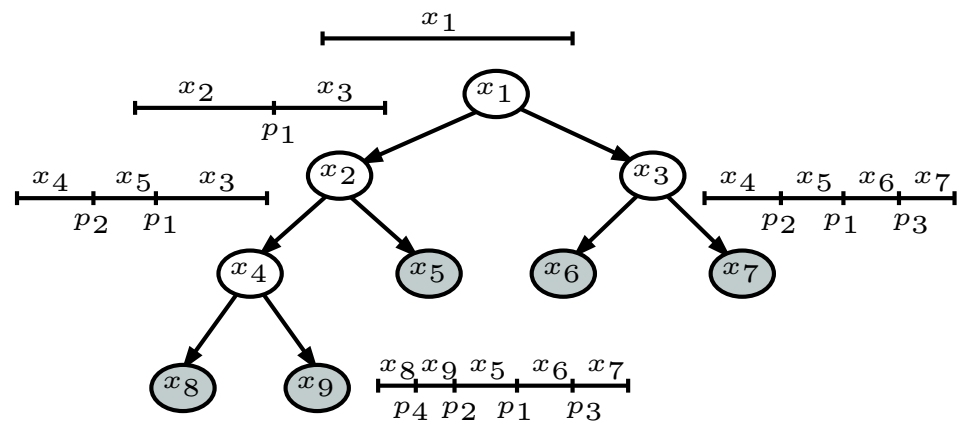

Fig. 1. Configuration tree for a point sequence $P=\left(p_{1}, p_{2}, p_{3}, p_{4}\right)$.

Therefore, the above strategy constructs eventual configuration tree, and then obtains the rough partition of the unit segment. So, each interval length corresponding a leaf is calculated using the magic number $y_{1}$. Since each inner node has exactly two children and both interval are known, every interval length is also determined in the direction from leaves toward root.

\subsection{Optimality and correctness}

Finally, we show that the maximum gap ratio $R_{P}$ of the point sequence $P$ computed by our strategy is equal to $R_{n}$. The magic number $y_{1}$ plays very important roles to optimize $R_{P}$, and so it is made neatly. How to determine $y_{1}$ and the optimality requires Lemma 3 . And Lemma 4 shows the correctness of the strategy.

Lemma 3. If any set of intervals $\left\{x_{i+1}, \ldots, x_{2 i+2}, x_{2 i+3}\right\}$ are sorted in non-increasing order with respect to their lengths, then the above strategy achieves the maximum gap ratio $R_{P}=2^{\lfloor n / 2\rfloor /(\lfloor n / 2\rfloor+1)}$.

Proof. Let $y_{i}$ denote the length of $x_{2(l+i)+1}$ for $i=0,1, \ldots, k$, where $k=\lfloor n / 2\rfloor$ and $l=\lceil n / 2\rceil$. Note that the node $x_{l}$ has interval $y_{1}$ as one of the children in the tree configuration. Now, we assume the gap ratio $r_{i}$ is defined by $\frac{x_{i+1}}{x_{2 i+1}}=\frac{x_{i+1}}{y_{i}}$ for $(l+i)$-th insertion. By Lemma 4, this definition for $r_{i}$ does not induce an inconsistency. From this fact, the minimum interval is $y_{i}$ and the maximum interval is $x_{2(l+i)}+x_{2(l+i)+1}=$ $2 y_{i+1}$, for $(l+i)$-th insertion $(1 \leq i<k)$. For the last insertion, the minimum interval is $y_{k+1}$ and the maximum interval is $y_{1}$. Therefore, the gap ratios $r_{i}$ for $i=l, l+1, \ldots, l+k$, are described as follow,

$$
\begin{aligned}
& r_{l}=\frac{x_{l+1}}{x_{2 l+1}}=\frac{x_{2 l+2}+x_{2 l+3}}{x_{2 l+1}}=\frac{2 y_{2}}{y_{1}}, \\
& r_{l+1}=\frac{x_{l+2}}{x_{2 l+3}}=\frac{x_{2 l+4}+x_{2 l+5}}{x_{2 l+3}}=\frac{2 y_{3}}{y_{2}}, \\
& r_{n-1}=r_{l+k-1}=\frac{x_{l+k}}{x_{2(l+k-1)+1}}=\frac{x_{2(l+k)}+x_{2(l+k)+1}}{x_{2(l+k-1)+1}}=\frac{2 y_{k+1}}{y_{k}}, \\
& r_{n}=r_{l+k}=\frac{x_{l+k+1}}{x_{2(l+k)+1}}=\frac{y_{1}}{y_{k+1}} .
\end{aligned}
$$


Since $x_{2 i+3}>x_{4 i+2}$ for $i \leq l-1, r_{i}=\frac{x_{i+1}}{x_{2 i+1}}=\frac{x_{2 i+2}+x_{2 i+3}}{x_{4 i+2}+x_{4 i+3}} \leq \frac{x_{2 i+2}}{x_{4 i+3}}=r_{2 i+1}$. This implies $R_{n}=\max \left\{r_{l}, r_{l+1}, \ldots, r_{n}\right\} \geq$ $\max \left\{r_{1}, r_{2}, \ldots, r_{l-1}\right\}$. Thus, $R_{n}$ is minimized when

$$
\begin{aligned}
R_{n} & =\left(r_{l} \cdot r_{l+1} \cdots \cdots r_{l+k}\right)^{\frac{1}{k+1}} \\
& =\left(\frac{2 y_{2}}{y_{1}} \cdot \frac{2 y_{3}}{y_{2}} \cdots \cdots \frac{2 y_{k+1}}{y_{k}} \cdot \frac{y_{1}}{y_{k+1}}\right)^{\frac{1}{k+1}}=2^{\frac{k}{k+1}}=2^{\left\lfloor\frac{n}{2}\right\rfloor /\left(\left\lfloor\frac{n}{2}\right\rfloor+1\right)} .
\end{aligned}
$$

Since every $r_{i}=R_{n}$, we have

$$
y_{i}=\frac{2}{R_{n}} y_{i+1}
$$

for $i=1, \ldots, k$, and

$$
y_{k+1}=\frac{y_{1}}{R_{n}} .
$$

Moreover, since

$$
y_{1}=\left(\frac{2}{R_{n}}\right)^{i-1} y_{i+1}
$$

for $i=2, \ldots, k+1$, if $y_{1}$ can be determined then every $y_{i}$ is also determined. When $n$ is odd, we have

$$
y_{1}=\frac{1}{2 \sum_{j=1}^{k+1}\left(\frac{R_{n}}{2}\right)^{j-1}}
$$

since

$$
1=\sum_{i=n+1}^{2 n+1} x_{i}=2 \sum_{j=1}^{k+1} y_{j}=2 \sum_{j=1}^{k+1}\left(\frac{R_{n}}{2}\right)^{j-1} .
$$

Similarly, when $n$ is even, we have

$$
y_{1}=\frac{1}{1+2 \sum_{j=2}^{k+1}\left(\frac{R_{n}}{2}\right)^{j-1}}
$$

since

$$
1=\sum_{i=n+1}^{2 n+1} x_{i}=y_{1}+2 \sum_{j=2}^{k+1} y_{j}
$$

Observation 2. $y_{1} \geq y_{2} \geq \cdots \geq y_{k+1}$.

The observation follows from the facts that $y_{i}=\frac{2}{R_{n}} y_{i+1}$ and $\frac{2}{R_{n}}$ is greater than 1 .

In order to show the correctness of this strategy and the optimality, we have to prove that the interval sequence $\left(x_{i+1}, \ldots, x_{2 i}, x_{2 i+1}\right)$ generated by $p_{i}$ is sorted in non-increasing order with respect to their length for every $1 \leq i \leq n$.

Lemma 4. Whenever our strategy partitions the interval $x_{i}$ for every $1 \leq i \leq n$, the resulting interval sequence $\left(x_{i+1}, x_{i+2}, \ldots, x_{2 i_{1}}\right)$ is sorted in non-increasing order, that is, inequalities $x_{i+1} \geq x_{i+2} \geq \cdots \geq$ $x_{2 i} \geq x_{2 i+1}$ are satisfied. 


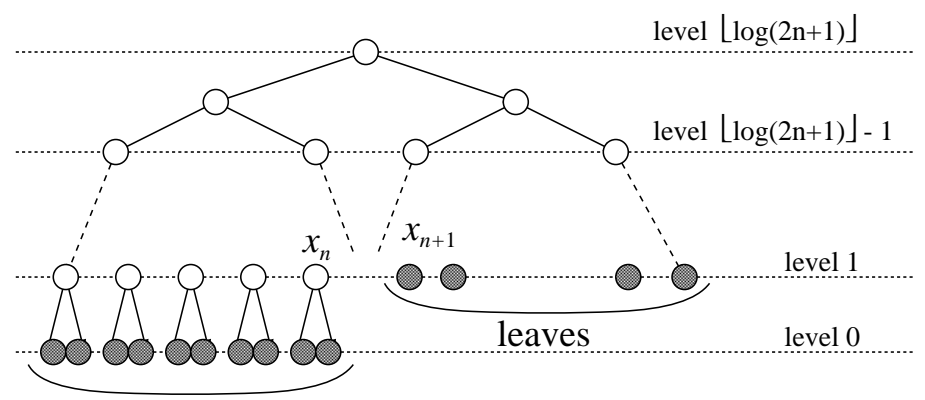

leaves

Fig. 2. The levels of tree corresponding the behavior of our strategy.

Proof. It can be proved by induction on level of the tree configuration with size $2 n+1$. The level of a node $v$ is defined as $\lceil\log (2 n+1)\rceil-$ the height of $v$. So, all leaf nodes may be in the level 0 or 1 , and the level of root $x_{1}$ is $\lfloor\log (2 n+1)\rfloor$, (see Figure 2).

When $2^{h}=n+1$, where $h=\lfloor\log (2 n+1)\rfloor$, all leaf nodes are in level 0. In this case, from Observation 2, the statement $x_{n+1} \geq \ldots \geq x_{2 n+1}$ is hold. When $n+1 \neq 2^{h}$, the intervals $x_{n}$ and $x_{n+1}$ are in same level 1 . Then,

$$
x_{n}=2 y_{k+1}=2\left(\frac{R_{n}}{2}\right)^{k} y_{1}=\frac{R_{n}^{k+1}}{R_{n} 2^{k-1}} y_{1}=\frac{2^{k}}{R_{n} 2^{k-1}} y_{1}=\frac{2}{R_{n}} y_{1}>y_{1}=x_{n+1} .
$$

On the remaining nodes in level 1 , the both children of a node are the intervals which has a same length so as $2 y_{j}$. For the consequence two intervals $x_{i}$ and $x_{i+1}, x_{i} \geq x_{i+1}$ by Observation 2.

Let $I^{i}=\left(z_{1}^{i}, \ldots, z_{2^{i}}^{i}\right)$ be the intervals in level $i$, where $z_{1}^{i}$ and $z_{2^{i}}^{i}$ are the leftmost and rightmost intervals in level $i$, respectively. Now, we assume that the statement is hold up to level $i$, that is, $z_{1}^{i} \geq z_{2}^{i} \geq \cdots \geq z_{2^{i}}^{i} \geq$ $\cdots \geq x_{2 n+1}$. By this induction hypothesis, $z_{2^{i-1}}^{i-1}=z_{2^{i}-1}^{i}+z_{2^{i}}^{i} \geq z_{1}^{i+1}+z_{2}^{i+1}=z_{1}$. For the others, $z_{j}^{i-1} \geq z_{j+1}^{i-1}$ are hold by similar arguments in the base step.

Thus, we have a conclusion on 1-dimensional dispersion problem.

Theorem 1. Given an integer n, our strategy gives an optimal solution with the maximum gap ratio being $2^{\frac{\lfloor n / 2\rfloor}{[n / 2]+1}}$ on 1-dimensional dispersion problem in $O(n)$ time.

\section{2-dimensional problem}

\subsection{Notations and analytical results}

Let $s_{1}=(0,0), s_{2}=(1,0), s_{3}=(1,1)$, and $s_{4}=(0,1)$ be the four corner points of $\mathbb{S}^{2}$. For each point set $S_{i}$ after inserting $i$ points in $P$, we define two empty circles $C_{i}$ and $c_{i}$ : The diameter of $C_{i}$ is $G_{i}$ and the center $p$ of $C_{i}$ satisfies $\min _{s \in S_{i}} d(s, p)=G_{i} / 2$. The diameter of $c_{i}$ is $g_{i}$ and its center is the midpoint of the closest pair of points. Note that the two empty circles are not unique, since the maximum gap and the minimum one may be defined by some of the triples or pairs. We break ties arbitrarily to choose $C_{i}$ and $c_{i}$. For any three different points $p_{1}, p_{2}$, and $p_{3}$, not on a line, let $C\left(p_{1}, p_{2}, p_{3}\right)$ be the circle passing through the three points. The interior of a circle $C$ is denoted by int $C$ and the diameter of $C$ is denoted by $\operatorname{diam}(C)$. The gap ratio $r_{i}$ is defined by $r_{i}=G_{i} / g_{i}=\operatorname{diam}\left(C_{i}\right) / \operatorname{diam}\left(c_{i}\right)$.

Lemma 5. For $i=1,2, \ldots, n-1$, if $\max _{1 \leq j \leq i} r_{j}<2$, then $p_{i+1}$ must be inserted in int $C_{i}$, to keep $r_{i+1}<2$.

Proof. If $p_{i+1}$ does not lie in int $C_{i}$, then $G_{i+1}=G_{i}$. On the other hand, $g_{i+1} \leq G_{i} / 2$, since there is no empty circle whose diameter is greater than that of the largest empty circle. Hence $r_{i+1} \geq 2$. 
Fact 1. For two acute triangles $\triangle A B C$ and $\triangle D E F$, if $\angle A B C \leq \angle D E F$ and $\angle B C A \geq \angle E F D$, then

$$
\frac{|A B|}{|C A|} \geq \frac{|D E|}{|F D|}
$$

We have equality if $\angle A B C=\angle D E F$ and $\angle B C A=\angle E F D$. See Fig. 3 for an example.

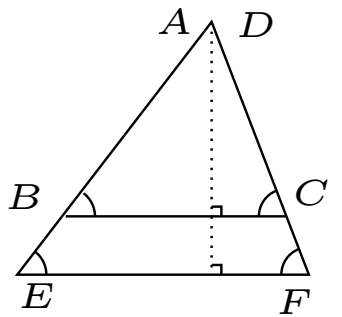

$A B: D E=A C: D F$

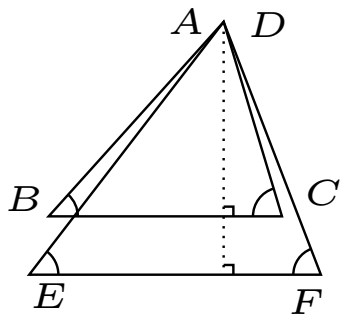

$A B: D E \geq A C: D F$

Fig. 3. Illustration for Fact 1

Lemma 6. The last point $p_{n}$ must lie at the center of $C_{n-1}$ to minimize the maximum gap ratio.

Proof. By Lemma 5, we assume that $p_{n}$ is inserted into the interior of $C_{n-1}$. If there exists an empty circle with diameter equal to $G_{n-1}$, then this lemma immediately holds, since it must maximize the minimum gap $g_{n}$.

We assume that $C_{n-1}$ passes through three points $a, b$ and $c$ in the counter-clockwise order. Let $C^{\prime}$ be the other empty circle (not $C_{n}$ ) passing through $a$ and $b$.

We move $p_{n}$ along the perpendicular bisector of $a$ and $b$ so as to decrease $g_{n}$. Note that we may also have to consider motions between $b$ and $c$, and between $c$ and $a$. But similar arguments can be appropriately applied.

In this situation, a pair of points defining $g_{n}$ is never changed, i.e., $g_{n}=d\left(p_{n}, a\right)=d\left(p_{n}, b\right)$. However, a triple (or pair) of points defining $G_{n}$ may change. There are two kinds of meaningful circles which may define $G_{n}$; the first one is $C^{\prime}$ defined above and the second one is the empty circle $C^{\prime \prime}$ that passes through $a$ and $p_{n}$. The other circles may lead to $r_{n} \geq 2$, or may not lead to a better $r_{n}$ than that of $C^{\prime}$ or $C^{\prime \prime}$. Note that $p_{n}$ has to be inserted at the center of $C_{n-1}$ to maximize $g_{n}$, when $C_{n}=C^{\prime}$. On the other hand, when $C_{n} \neq C^{\prime}, p_{n}$ lies in int $C^{\prime}$.

Now, we assume $C_{n}=C^{\prime \prime}$. Let $o$ be the center of $C_{n-1}, o_{1}$ be the center of $C^{\prime}$ and $o_{2}$ be the center of $C^{\prime \prime}$. Consider two triangles $\Delta_{1}=\Delta\left(a, o_{2}, p_{n}\right)$ and $\Delta_{2}=\Delta\left(a, o_{1}, o\right)$. Since $p_{n}$ is in $C^{\prime}$, it can be seen that $\angle a o_{2} p_{n}<\angle a o_{1} O$ and $\angle a p_{n} o_{2}>\angle a o o_{1}$ by simple calculations. Hence, we have $d\left(a, o_{2}\right) / d\left(a, p_{n}\right)>$ $d\left(a, o_{1}\right) / d(a, o)$ from Fact 1 . This concludes the proof.

By Lemma 6, we can find an optimal 2-point sequence. Fig.4 shows the notations for an instance of $n=2$. In this case, there are three circles, but the meaningful circles are just $C^{\prime}$ and $C^{\prime \prime}$ from Lemma 6 . Hence, $p_{2}$ is put at the center of $C_{1}$.

We can assume that $p_{1}$ lies on the line $y=\frac{1}{2}$, to maximize $g_{1}$. Since we can specify $p_{2}$ once $p_{1}$ is determined, we only examine an $x$-coordinate of $p_{1}$. The maximum gap ratio $R_{P}$ is minimized when $g_{1}=$ $g_{2}$, and then an optimal point pair satiafies $G_{1}^{2}=2 g_{1} G_{2}$, by simple observations. Hence, for example, 


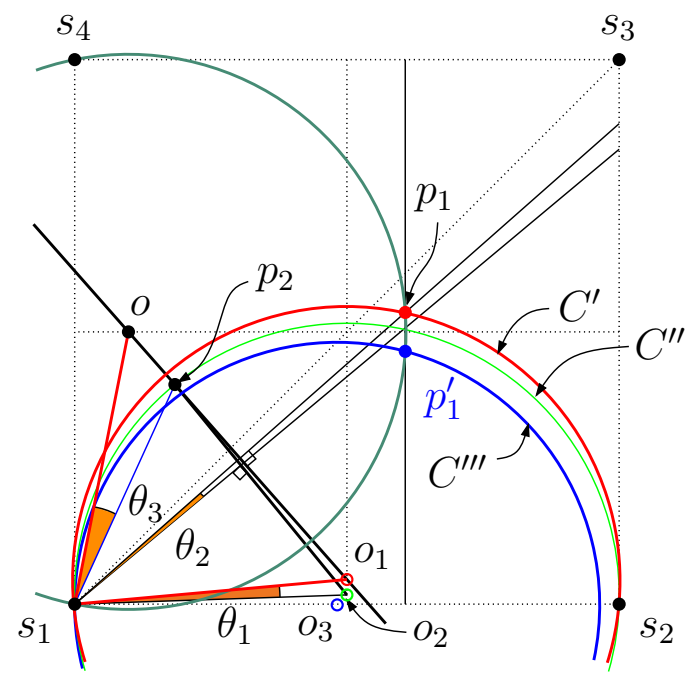

Fig. 4. Notations of Lemma 6 for an instance of $n=2$.

these gaps $G_{1}, g_{1}$ and $G_{2}$ are given by

$$
\begin{aligned}
G_{1} & =\frac{4 x_{1}^{2}-8 x_{1}+5}{4-4 x_{1}}, \\
g_{1} & =\sqrt{x_{1}^{2}+\frac{1}{4}}, \\
G_{2} & =2 \sqrt{\frac{1}{4}+\left(x_{1}-\frac{1}{2}\right)^{4}} .
\end{aligned}
$$

Solving this simultaneous equations, we obtain the coordinates of optimal points;

$$
\begin{aligned}
& p_{1}^{*}=(0.273704,0.5), \\
& p_{2}^{*}=(0.808958,0.5) .
\end{aligned}
$$

Next, we consider the cases of $n=3$ and larger $n$. They are more complicated and may not be solvable in an analytical sense.

Lemma 7. If $n \geq 3$ and the first point $p_{1}$ lies on the line $y=\frac{1}{2}$ or the line $x=\frac{1}{2}$, then the maximum gap ratio is greater than or equal to 2.

Proof. We assume that the first point $p_{1}$ lies on the line $y=\frac{1}{2}$. When $p_{2}$ is inserted into int $C_{1} \cap$ int $C\left(p_{1}, s_{1}, s_{2}\right), p_{3}$ should be inserted at the center of $C\left(p_{1}, s_{3}, s_{4}\right)$ from Lemma 6 , and then $C_{3}$ is defined by $C\left(p_{2}, s_{1}, s_{2}\right)$. Since $\operatorname{diam}\left(C_{3}\right) \leq 1$ and $\operatorname{diam}\left(C\left(p_{1}, s_{3}, s_{4}\right)\right) \geq 1, r_{3} \geq 2$. So, $p_{2}$ has to be inserted anywhere in int $C_{1} \backslash$ int $C\left(p_{1}, s_{1}, s_{2}\right) \cap \mathbb{S}^{2}$. Hence, $C_{2}$ is defined as the circle passing through $p_{1}, s_{1}$ and $s_{2}$. By Lemma $5, p_{3}$ is inserted into the interior of $C_{2}$. Therefore, the third gap ratio $r_{3}$ is at least 2 , since $\operatorname{diam}\left(C_{2}\right)=\operatorname{diam}\left(C_{3}\right)$ and $\operatorname{diam}\left(c_{2}\right) \leq \frac{1}{2} \operatorname{diam}\left(C_{2}\right)$.

By the symmetry, the similar argument can be applied when $p_{1}$ lies on the line $x=\frac{1}{2}$.

Lemma 8. When $n=3$, the second point $p_{2}$ should be inserted at the center of $C_{1}$.

Proof. Let $C_{1}^{\prime}$ and $C_{1}^{\prime \prime}$ be the second and third largest empty circles of $S_{1}$. Consider a case in which there exist exactly two circles, $C_{1}$ and $C_{1}^{\prime}$, with their diameters greater than 1 , at the end of the first insertion. Then, $p_{2}$ must be in int $C_{1}^{\prime} \cap\left\{p \in S \mid d\left(p_{1}, p\right)>\frac{1}{2}\right\} \cap\left\{p \in S \mid d\left(s_{i}, p\right)>\frac{1}{2}\right\}$, where $s_{i}$ is the nearest corner 
point of $S$, since $C_{2}$ passes $p_{2}, \operatorname{diam}\left(C_{2}\right)>1$ and $g_{2}=\max \left\{d\left(p_{1}, p_{2}\right), d\left(s_{i}, p_{2}\right)\right\}<\frac{1}{2}$. If such intersection does not exist, then we can see $R_{P} \geq 2$. Let $x$ and $x^{\prime}$ be the centers of $C_{1}$ and $C_{1}^{\prime}$, respectively. Since $p_{2}$ is inserted in that intersection, $C_{2}=C\left(p_{2}, s_{i}, s_{j}\right)$, where $s_{j}$ is the second nearest corner point of $\mathbb{S}^{2}$ from $p_{2}$. Let $x^{\prime \prime}$ be the center of $C_{2}$. Now, consider two triangles, $\Delta s_{i} x^{\prime \prime} p_{2}$ and $\Delta s_{i} x^{\prime} x$. From Fact $1, p_{2}$ is inserted at the center of $C_{1}$, to minimize $r_{2}$.

Next, consider the next case that there are three empty circles, $C_{1}, C_{1}^{\prime}$, and $C_{1}^{\prime \prime}$, with diameters greater than 1, at the end of the first insertion. This case occurrs when $p_{1}$ is contained in exactly one circle $C\left(o, s_{i}, s_{j}\right)$, where $o$ is the center, $\left(\frac{1}{2}, \frac{1}{2}\right)$ and $s_{i}$ and $s_{j}$ are corner points of $S$. We assume that $p_{2}$ is inserted in int $C_{1}^{\prime} \cap\left\{p \in S \mid d\left(p_{1}, p\right)>\frac{1}{2} \operatorname{diam}\left(C_{1}^{\prime \prime}\right)\right\} \cap\left\{p \in S \mid d\left(s_{i}, p\right)>\frac{1}{2} \operatorname{diam}\left(C_{1}^{\prime \prime}\right)\right\}$, where $s_{i}$ is the nearest corner point of $S$ from $p_{2}$, the maximum gap ratio may be less than 2 . If the intersection does not exist, then $R_{P} \geq 2$. However, the same argument as above applies. Therefore, $p_{2}$ should be inserted at the center of $C_{1}$, if $n=3$.

Lemma 9. When $n=3$, all largest empty circles, $C_{1}, C_{2}$ and $C_{3}$, pass through $p_{1}$.

Proof. It is obvious that $C_{1}$ and $C_{2}$ pass through $p_{1}$ from Lemmas 7 and 8 . If $p_{2}$ lies on the line $y=\frac{1}{2}$, then $p_{3}$ lies on the line $x=\frac{1}{2}$, and vice versa. We can assume that $p_{2}$ and $p_{3}$ are contained on the boundary of the square $\left[0, \frac{1}{2}\right] \times\left[0, \frac{1}{2}\right]$ from Lemmas 4 and 8 . Then, $p_{1}$ lies in the (open) square $\left(\frac{1}{2}, 1\right) \times\left(\frac{1}{2}, 1\right)$. Hence, $C_{3}$ passes through $p_{1}$, since the half space $y>\frac{1}{2}$ contains $p_{1}$ but not $p_{2}$ or $p_{3}$.

Since $p_{2}$ and $p_{3}$ are inserted at center $\left(C_{1}\right)$ and center $\left(C_{2}\right)$, respectively, we obtain $g_{2}=\frac{1}{2} G_{1}$ and $g_{3}=\frac{1}{2} G_{2}$. In order to minimize $R_{P}$, we take geometric average among $r_{1}, r_{2}$ and $r_{3}$;

$$
R_{P}=\sqrt[3]{r_{1} \cdot r_{2} \cdot r_{3}}=\sqrt[3]{\frac{G_{1}}{g_{1}} \frac{G_{2}}{g_{2}} \frac{G_{3}}{g_{3}}}=\sqrt[3]{\frac{G_{1}}{g_{1}} \frac{G_{2}}{\frac{1}{2} G_{1}} \frac{G_{3}}{\frac{1}{2} G_{2}}}=\sqrt[3]{2^{2} \frac{G_{3}}{g_{1}}}
$$

Hence, if we can show $\frac{G_{3}}{g_{1}}<2$, then $R_{p}<2$ is obtained when $n=3$. However, it seems to be difficult to specify an optimal position of $p_{1}$ satisfying the above condition, and then solving analytically the exact positions in an optimal point sequence is too complicated even in the case of $n=3$. Then, we propose a heuristic algorithm for finding a good point sequence.

\subsection{Heuristic Algorithms}

We present a simple heuristic algorithm based on local search. First, we describe a procedure to compute the maximum gap ratio, for given a $n$-point sequence $P$. Then, we show a main procedure which treats $n$-point sequence $\left(p_{1}, \ldots, p_{n}\right)$ as a point $\left(x_{1}, y_{1}, x_{2}, y_{2}, \ldots, x_{n}, y_{n}\right)$ in the $2 n$-dimensional space $\mathbb{R}^{2 n}$ to find a best point by examining its neighborhood in $\mathbb{R}^{2 n}$. This technique is similar to the lifting technique common in computational geometry.

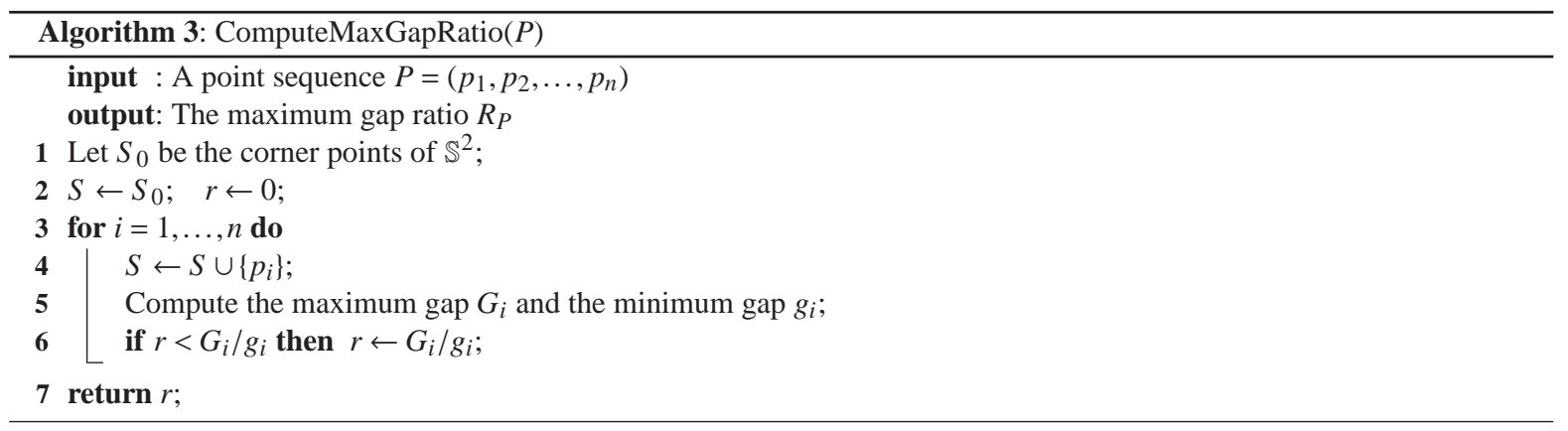

Algorithm 3 computes the maximum gap ratio for a given $n$-point sequence. It runs in $O\left(n^{2}\right)$ time. In particular, we maintain a planar subdivision by Delaunay triangulation [15] for each $S_{i}$. The planar 
subdivision by Delaunay triangulation is a planar graph, and then each face contributes to an empty circle and each edge represents the neighborhood relation between two connecting vertices (see [16]). Hence, we obtain the gaps $G_{i}$ and $g_{i}$ in linear time, since the reconstruction of the subdivision is the crucial part.

Algorithm 4 is a main procedure of our heuristics. Given three parameter $n, m$ and $k$, the algorithm iterates local search $k$ times starting from randomly generated point sequences. In each iteration we compute a local optima of $n$-point sequence. The parameter $m$ is used to specify a termination condition to guarantee the accuracy of solutions obtained.

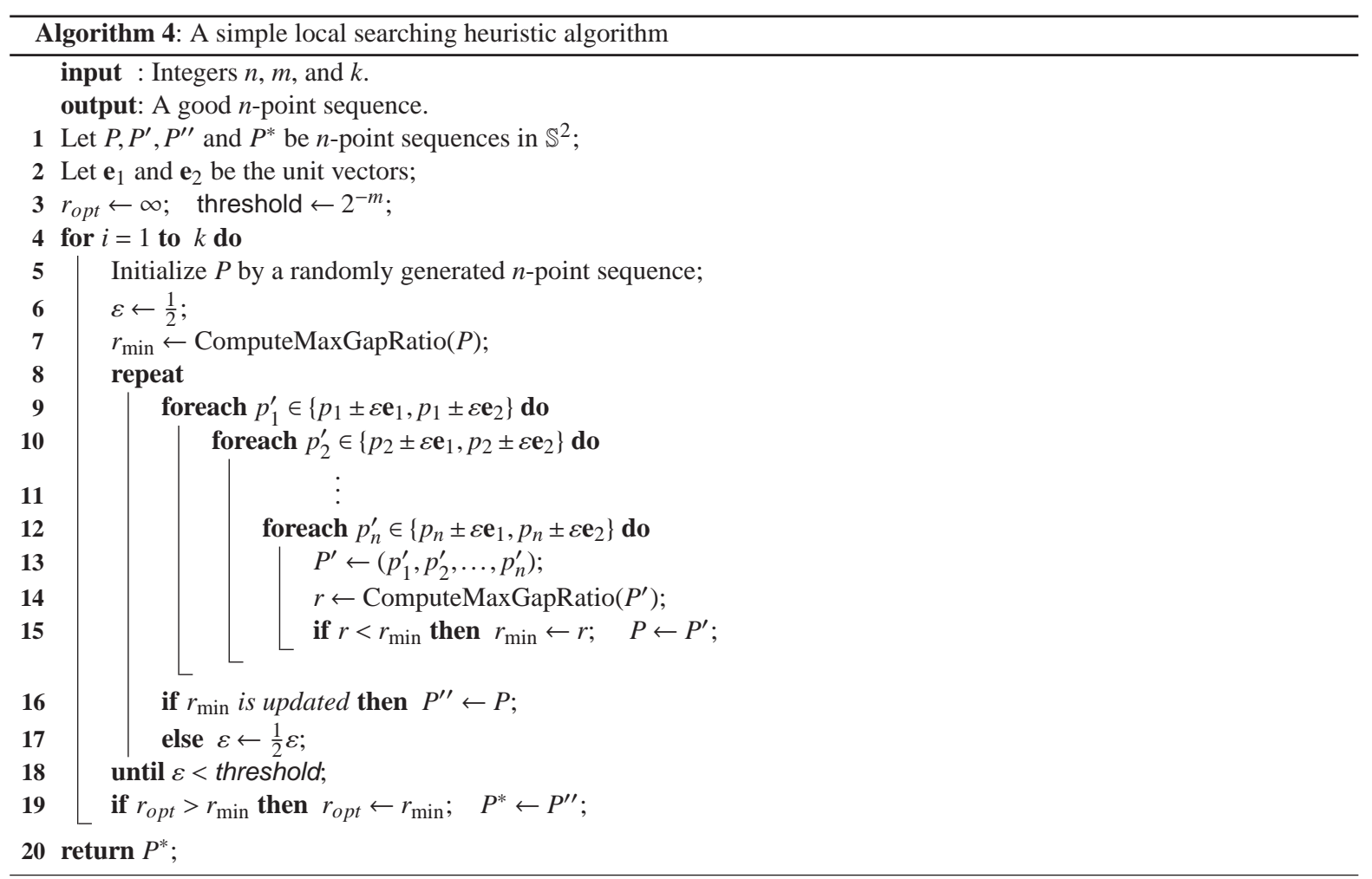

\subsection{Experimental Results}

We have implemented Algorithm 4 to evaluate the accuracy of the solutions obtained by the heuristic algorithm. Table 1 describes our environment of the experiment. We designed the algorithm using the exact computation in LEDA[10] for the sake of accuracy and for robustness.

Table 1. The environment of experiment

\begin{tabular}{|c|c|c|}
\hline Workstation & $\mathrm{CPU}$ & Main memory \\
\hline Dell PowerEdge SC1425 Server & 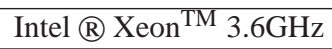 & $8 \mathrm{~GB}$ \\
\hline$\overline{\mathrm{OS}}$ & Compiler & External library \\
\hline RedHat Enterprise Linux 3 & $\mathrm{~g}++-3.4 .2$ & LEDA-5.0.1 \\
\hline
\end{tabular}

Table 2 shows the best $R_{p}$ values obtained by Algorithm 4 . For each $n=2,3,4,5$, we executed the algorithm more than 1000 times with the threshold less than $10^{-8}$. For each of $n=6,7,8$, we executed with 500, 100, and 20 trials with the same accuracy. 


\begin{tabular}{|c||c|c|c|c|c|c|}
\hline$n$ & 2 & 3 & 4 & 5 & 6 & 7 \\
\hline$R_{p}$ & 1.87804 & 1.92716 & 1.927164 & 1.92716 & 1.927203 & 1.99312 \\
\hline
\end{tabular}

As mentioned above, we have an exact value of $R_{2}$ and a property for $R_{3}$. The computed value $R_{2}$ shown in the table finds to be close enough to $R_{2}$. The 3-point sequence achieving the computed value of $R_{3}$ shown in the table satisfies the property $\frac{G_{3}}{g_{1}}<2$, and we conjecture that the point sequence is optimal. In addition to this, the obtained point sequences for $n=4,5,6$ may also be optimal, since these maximum gap ratios are roughly the same.

There is a gap between the results for $n=6$ and $n=7$. We have obtained a better sequence than that of Voronoi insertion. However, in the case of $n=8$, we did not obtain a sequence with the maximum gap ratio less than 2. In our environment of experiment, we gave up to apply the algorithm for $n \geq 8$, since it is too slow. In fact, it took one day per one trial.

We could use those point sequences obtained above as seed point sequences and perform the incremental Voronoi insertion.

Table 3. A good seed point sequence and the initial maximum gap ratio.

\begin{tabular}{|c|c|c|c|}
\hline$p_{1}$ & $p_{2}$ & $p_{3}$ & $p_{4}$ \\
\hline$(0.769146,0.501913)$ & $(0.263398,0.508807)$ & $(0.499994,0.0637435)$ & $(0.477718,0.891089)$ \\
\hline \hline$p_{5}$ & $p_{6}$ & $p_{7}$ & $R_{P}$ \\
\hline$(2.0687 e-05,0.317322)$ & $(8.21674 e-06,0.662797)$ & $(0.999993,0.304037)$ & 1.993124 \\
\hline
\end{tabular}

We have implemented the above-stated strategy using the 7-point sequence shown in Table 3 as a starting seed point sequence. The initial maximum gap ratio is 1.993124 . Fig.5 indicates the resulting point distribution. The maximum gap ratio of this point sequence is actually 1.99921 .

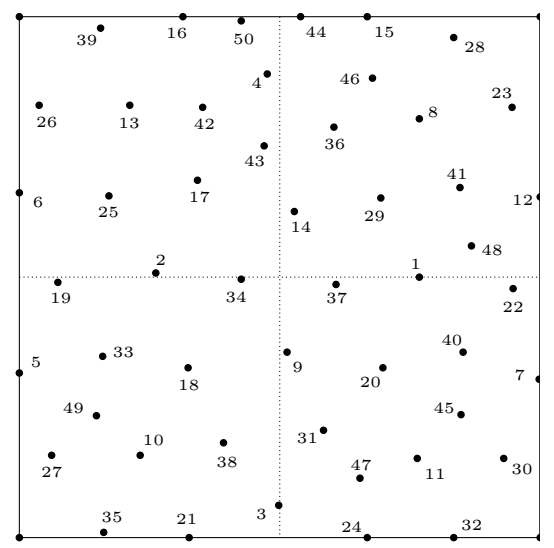

Fig. 5. A good 50-point sequence with the maximum gap ratio bounded by 1.99921 .

Furthermore, we consider the irregularity of the final point distribution for each of our results and Voronoi insertion. In order to enhance the difference between them, We use a Delaunay triangulations shown in Fig. 6. Our distribution is pretty irregular, compared with that obtained by Voronoi insertion.

One of the notable remarks is that Voronoi insertion easily gives a uniform point sequence in our criteria, but the final distribution is a totally unfair and locally regular distribution. Performing a per- 


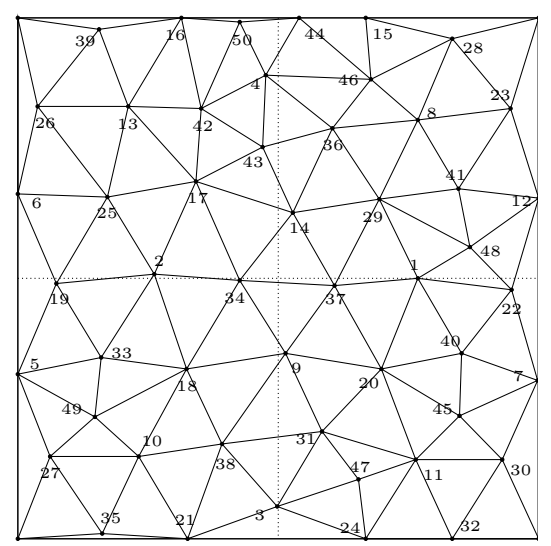

Fig. 6. The Delaunay triangulations for the resulting point distribution for our 50-point sequence with the maximum gap ratio bounded by 1.99921 and the incremental Voronoi insertion.

turbation to fix the badness, our criteria immediately is evaluated as the modified point sequence is not uniform. Therefore, our approach is one of the solutions which overcome this dilemma.

\section{Conclusions}

In this paper we have presented a preliminary result on dispersion. One of the most important future works is to extend the result to higher dimensions. We had some results on lower and upper bounds of the maximum gap ratio for the planar case, but none in the higher dimensions.

\section{Acknowledgments}

The first two authors would like to thank Ryuhei Uehara and Taisuke Shimamoto for reading the first version of the manuscript. This work was partially supported by the Ministry of Education, Science, Sports and Culture, Grant-in-Aid for Scientific Research on Priority Areas and Scientific Research (B).

\section{References}

1. B. Aronov, T. Asano, Y. Kikuchi, S. C. Nandy, S. Sasahara, and T. Uno, ”A Generalization of Magic Squares with Applications to Digital Halftoning," to appear in Theory of Computing System.

2. T. Asano, "Computational Geometric and Combinatorial Approaches to Digital Halftoning," Proc. of Conferences in Research and Practice in Information Technology, 51, p.3, 2006.

3. T. Asano, P. Brass, and S. Sasahara, "Disc Covering Problem with Application to Digital Halftoning," Proc. Int. Conf. on Computer Science and Applications, vol.3, pp.11-21, 2004.

4. T. Asano, N. Katoh, K. Obokata, and T. Tokuyama, "Matrix rounding under the $L_{p}$-discrepancy measure and its application to digital halftoning, SIAM J. Computing, 32, 6, pp.1423-1435, 2003.

5. B. Chazelle: "The Discrepancy Method: Randomness and Complexity," Cambridge University Press, 2000.

6. C. R. Collins and K. Stephenson, "A Circle Packing Algorithm," Comput. Geom., Theory and Applications, 25, 3, pp.233-256, 2003.

7. B. Doerr, Nonindependent Randomized Rounding and an Application to Digital Halftoning," SIAM Journal on Computing, 34, 2, pp.299-317, 2005.

8. S. Gooran, "Context Dependent Colour Halftoning in Digital Printing," Department of Science and Technology, Linkoping University, Sweden Proceedings of the Conference on Image Processing, Image Quality, Image Capture Systems (PICS-00), pp.242-246, 2000.

9. J. Matoušek: "Geometric Discrepancy," Springer, 1991.

10. K. Mehlhorn and S. Näher: "LEDA - A Platform of Combinatorial and Geometric Computing," Cambridge University Press, Cambridge, England, 1999. 
11. T. Mitsa and K. J. Parker, Digital Halftoning using a Blue-Noise Mask," Journal of the Optical Society of America A, 9 , 11, pp.1920-1929, 1992.

12. K. J. Nurmela, P. R. J. Östergård and R. aus dem Spring, "Asymptotic Behavior of Optimal Circle Packings in a Square," Canadian Mathematical Bulletin, 42, 3, pp.380-385, 1999.

13. K. J. Nurmela and P. R. J. Östergård, ”Packing up to 50 Equal Circles in a Square,” Discrete and Computational Geometry, 18, 1, pp.111-120, 1997.

14. K. J. Nurmela, ”More Optimal Packings of Equal Circles in a Square,” Discrete and Computational Geometry, 22, 3 , pp.439-457, 1999.

15. A. Okabe, B. Boots, K. Sugihara, and S.N. Chiu: "Spatial Tessellations: Concepts and Applications of Voronoi Diagrams," John Wiley \& Sons, 2000.

16. F.P. Preparata and M.I. Shamos: "Computational Geometry: An Introduction," Springer-Verlag, 1985.

17. K. Sadakane, N. Takki Chebihi, and T. Tokuyama, "Discrepancy-based digital halftoning: Automatic evaluation and optimization," Interdisciplinary Information Sciences, 8, 2, pp.219-234, 2002.

18. R. Ulichney, ’Digital Halftoning," MIT Press, 1987. 